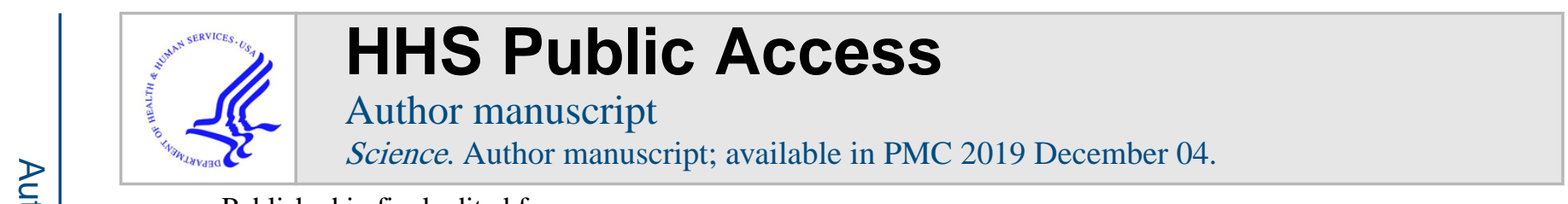

Published in final edited form as:

Science. 1978 June 23; 200(4348): 1399-1401. doi:10.1126/science.663620.

\title{
Quantitation of Hindered Rotations of Diphenylhexatriene in Lipid Bilayers by Differential Polarized Phase Fluorometry
}

\author{
J. R. Lakowicz, \\ Freshwater Biological Institute and Department of Biochemistry, University of Minnesota, Navarre \\ 55392
}

\author{
F. G. Prendergast \\ Department of Pharmacology, Mayo Medical School, Rochester, Minnesota 55901
}

\begin{abstract}
Diffusional motions of 1,6-diphenyl-1,3,5-hexatriene (DPH) were observed by diferential polarized phase fluorometry. The measurements indicated that the depolarizing rotations of DPH in propylene glycol are isotropic. The results in vesicles of dimyristoyl-L-a-phosphatidylcholine indicated that diffusional rotations of DPH are dominated by hindered torsional motions. Combined use of both differential phase and steady-state anisotropy measurements showed that the average rotational angle of DPH, at times long compared to the fluorescence lifetime, is limited to about $23^{\circ}$ at temperatures below the transition temperature of the lipid and that these rotations become less hindered above the transition temperature. The evidence that the depolarizing rotations of DPH in a lipid bilayer are different from those in an isotropic solvent calls into question the meaning of membrane microviscosity as determined by fluorescence anisotropy.
\end{abstract}

Measurements of the fluorescence polarization of probes embedded in lipid bilayers have been widely used to estimate the microviscosity of the hydrophobic regions of both natural and model cell membranes (1-6). In these microviscosity measurements it is assumed that the depolarizing rotational motions of the probe in lipid bilayers are equivalent to those in isotropic solvents.

1, 6-Diphenyl-1,3, 5-hexatriene (DPH) has become widely used in microviscosity studies because of its favorable fluorescence polarization and spectral properties (7), but perhaps more importantly because of the dramatic changes in fluorescence polarization that occur at the solid-liquid phase transition temperature of phospholipid bilayers $(8,9)$. These strongly temperature-dependent polarization changes are sharper with DPH than with other fluorophores, such as 12-anthroyl stearate, I-anilino-8-naph-thalenesulfonic acid, perylene, and $N$-phenyl-1-naphthylamine. A presumed advantage of DPH is the fact that the absorption and emission oscillators both lie along the long axis of the fluorophore. As a result, only rotations perpendiculart o this long axis will be effective in depolarizing the fluorescence emission. For these reasons, steady-state polarization measurements with DPH are considered to accurately describe the microviscosity of lipid bilayers.

Recent studies involving time-resolved decays of fluorescence anisotropy have demonstrated that DPH undergoes only hindered torsional motions below the phase transition temperature 
of dimyristoyl-L- $a$-phosphatidylcholine (DMPC) vesicles and that there is a highly nonexponential decay of anisotropy above the transition temperature (10). These phenomena reflect a hindered environment for DPH in lipid bilayers and illustrate the need to understand the types of probe motion that are responsible for fluorescence depolarization so that polarization data can be interpreted in terms of the membrane microviscosity.

In the work reported here we used differential polarized phase fluorometry to investigate the rotational diffusion of DPH in an isotropic solvent and in DMPC vesicles. This technique was used recently $(11,12)$ to study the rotational rates of fluorophores in isotropic solutions. Differences in the phase angle of the vertical and horizontal components of the emitted light are measured when the fluorescence sample is irradiated with polarized, sinusoidally modulated light. The tangent of the phase difference between the vertical and horizontal components of the fluorescence emission $(\tan \Delta)$ is a function of the fluorescence lifetime $(\tau)$, the fluorescence anisotropy (13) value in the absence of rotational diffusion $\left(r_{0}\right)$, the rotational rate in radians per second $(R)$, and the circular modulation frequency $(\omega)$, as shown by Eq. 1

$$
\tan \Delta=\frac{(2 R \tau) \omega \tau r_{0}}{m\left(1+\omega^{2} \tau^{2}\right)+(2 R \tau / 3)\left(2+r_{0}\right)+(2 R \tau)^{2}}
$$

where

$$
m=\frac{1}{9}\left(1+2 r_{0}\right)\left(1-r_{0}\right)
$$

The quadratic form of Eq. 1 may be used to obtain $\mathrm{R}$ from measurements of $\tan \Delta$.

$$
(2 R \tau)^{2}+(2 R \tau)\left(\frac{2+r_{0}}{3}-\left|\frac{r_{0}}{\tan \Delta}\right| \omega \tau\right)+m\left(1+\omega^{2} \tau^{2}\right)=0
$$

For isotropic rotations the maximum value possible for $\tan \Delta\left(\tan \Delta_{\max }\right)$ is a function of only $r_{0}, \omega$, and $\tau$.

$$
\tan \Delta_{\max }=\frac{3 \omega \tau r_{0}}{\left(2+r_{0}\right)+6\left[m\left(1+\omega^{2} \tau^{2}\right)\right]^{1 / 2}}
$$

Equations 1 to 4 apply to spherical molecules or isotropic rotators. If the rotational motions are isotropic the $\tan \Delta_{\max }$ values will agree with that predicted by Eq. 4. Anisotropic rotations, a heterogeneous population of isotropic rotators, or hindered torsional motions result in a $\tan \Delta_{\max }$ less than that predicted by Eq. 4. Studies of Mantulin and Weber (12) demonstrated the usefulness of differential polarized phase fluorometry in the detection of anisotropic rotations.

Figure 1 shows the fluorescence lifetimes and differential phase lifetimes of DPH in propylene glycol $(\omega) \Delta \tau=\tan \Delta)$. The precise agreement of the maximum value of the differential tangent with the theoretical value (Table 1) demonstrates that the depolarizing 
rotations of DPH in propylene glycol are isotropic. Additional evidence for this is provided by the precise agreement between the rotational rates calculated from the differential phase measurements and those calculated from the steady-state polarization measurements using the Perrin-Weber equation (Fig. 2; see the legend of Table 1 for a description of the experimental procedures).

Figure 3 shows the differential polarized phase measurements of DPH-la-beled DMPC vesicles. Similar results were obtained with unsonicated liposomes (14). These results demonstrate an increase in the rotational or torsional motions of DPH at the phase transition temperatures, but in contrast to the results with DPH in propylene glycol the maximum differential tangent is only 57 percent of the theoretical value predicted by Eq. 4 . This 43 percent tangent defect is greater than that predicted by Mantulin and Weber (12) for any degree of rotational anisotropy in the depolarizing motions. Since the depolarizing rotations of DPH are likely to be isotropic (7) and a similar tangent defect was observed in unsonicated liposomes of DMPC (which provide a more homogeneous population of probe molecules), we concluded that the tangent defect we observed was a result of hindered torsional motions of the probe when located in the lipid bilayer.

Recently, Weber (15) obtained solutions for the differential tangent under conditions where the rotational motions of the probe are limited to a nonzero anisotropy value $\left(r_{\infty}\right)$ at times long compared to $\tau$. Under these conditions the parallel $\left[I_{\|}(t)\right]$ and perpendicular $\left[I_{\perp}(t)\right]$ components of the fluorescence emission are given by

$$
\begin{gathered}
I_{\|}(t)=\left(1+2 r_{\infty}\right) \exp (-t / \tau)+2\left(r_{0}-r_{\infty}\right) \exp \left[-\left(\frac{1}{\tau}+6 R\right) t\right] \\
I_{\perp}(t)=\left(1-r_{\infty}\right) \exp (-t / \tau)-\left(r_{0}-r_{\infty}\right) \exp \left[-\left(\frac{1}{\tau}+6 R\right) t\right]
\end{gathered}
$$

From these equations Weber (15) obtained

$$
\begin{aligned}
& \tan \Delta=\omega \tau\left(r_{0}-r_{\infty}\right)(2 R \tau) /\left\{m\left(1+\omega^{2} \tau^{2}\right)+(2 R \tau / 3)\left[2+r_{0}-r_{\infty}\left(4 r_{0}-1\right)\right]\right. \\
& \left.+(2 R \tau)^{2}\left(1+2 r_{\infty}\right)\left(1-r_{\infty}\right)\right\}
\end{aligned}
$$

and

$$
\begin{aligned}
& \tan \Delta_{\max }=\omega \tau\left(r_{0}-r_{\infty}\right) /\left\{1 / 3\left[\left(2+r_{0}\right)-r_{\infty}\left(4 r_{0}-1\right)\right]\right. \\
& \left.+2\left[m\left(1+2 r_{\infty}\right)\left(1-r_{\infty}\right)\left(1+\omega^{2} \tau^{2}\right)\right]^{1 / 2}\right\}
\end{aligned}
$$

For $r_{\mathrm{\infty}}=0$, Eqs. 7 and 8 reduce to forms applicable to free isotropic rotations.

Since $r_{\infty}$ is a measure of the degree to which the depolarizing rotations of DPH are restricted, we wished to use our measurements to obtain $r_{\infty}$, in DMPC bilayers at various temperatures. Differential phase measurements alone do not yield $r_{\infty}$. However, in a hindered environment both the steady-state anisotropy and the differential tangent are functions of $r_{\infty}$, and $R$. With these measurements and the fluorescence lifetime it should be 
possible to determine $r_{\infty}$, and $R$. By integrating and normalizing Eqs. 5 and 6 over times from 0 to infinity we obtained

$$
r_{\infty}=r+\frac{\left(r-r_{0}\right)}{6 R \tau}
$$

Substituting Eq. 9 into Eq. 7 gives

$$
(C \tan \Delta)(2 R \tau)^{2}+(D \tan \Delta-A)(2 R \tau)+(E \tan \Delta-B)=0
$$

where

$$
\begin{aligned}
& A=3 B=\omega \tau\left(r_{0}-r\right) \\
& C=(1+2 r)(1-r) \\
& D=1 / 3\left(2 r-4 r^{2}+2\right) \\
& E=C / 9+m \omega^{2} \tau^{2}
\end{aligned}
$$

By measuring $\tan \Delta, r$, and $\tau$, one can obtain $R$ from Eq. 10. This value of $R$ may then be substituted into Eq. 9 to calculate $r_{\infty}$, . The values for $r_{\infty}$ so obtained are presented in Fig. 4 . It is apparent from these data that DPH is highly hindered at temperatures below the transition temperature of the bilayer $\left(r_{\infty} \simeq 0.3\right)$ and that DPH rotates freely above the transition temperature $\left(r_{\infty} \simeq 0\right)$. The midpoint of the change in $r_{\infty}$, occurs at $24^{\circ} \mathrm{C}$, which is near the phase transition temperature (8).

Using time-resolved decays of fluorescence anisotropy, Chen et al. (10) found $r_{\infty} \simeq 0.25$ below the transition temperature and $r_{\infty} \simeq 0.01$ above the transition temperature. Our results are in excellent agreement with those of Chen et al. However, the rapidity with which differential tangents may be measured allowed us to obtain a complete temperature profile for $r_{\infty}$ (Fig. 4). Thus differential phase fluoreometry will be a powerful method for investigating hindered motions of fluorophores in lipid bilayers.

The detection of restricted diffusional motions of DPH in lipid bilayers illustrates the difficulties inherent inr the extrapolation of DPH fluorescence anisotropy data to estimate membrane microviscosity. In such extrapolations it is assumed that the depolarizing rotations of DPH in the bilayers are identical to those in the reference solvent, which is usually a mixture of branched-chain alkanes. Our data demonstrate that this assumption is not valid, a result that calls into question the definition of membrane microviscosity from anisotropy measurements. Combined steady-state anisotropy measurements and differential phase fluorometry more accurately describe the rotational motions of probes in lipid bilayers and provide a better understanding of the constraints imposed by an anisotropic lipid bilayer. By such experiments it should also be possible to select fluorescence probes whose rotational motions in lipid bilayers are similar to those in homogeneous solutions, and thereby provide a better method for estimating membrane microviscosities.

\section{References and Notes}

1. Shinitzky M, Dianoux AC, Gilter C, Weber G, Biochemistry 10, 2106 (1971). [PubMed: 4104937] 
2. Cogan U, Shinitzky M, Weber G, Nishida T, ibid. 12, 521 (1973).

3. Brashford CL, Morgan CG, Radda GK, Biochim. Biophys. Acta 426, 157 (1976). [PubMed: 1252504]

4. Jacobson K and Wobschhall D, Chem. Phys. Lipids 12, 117 (1974). [PubMed: 4857064]

5. Moore NF, Barenholz Y, Wagner RR, J. Virol 19, 126 (1976). [PubMed: 985887]

6. Shinitzky M and Inbar M, J. Mol. Biol 85, 603 (1974). [PubMed: 4851371]

7. Shinitzky M and Barenholz Y, J. Biol. Chem 249, 2652 (1974). [PubMed: 4822508]

8. Lentz BR, Barenholz Y, Thompson TE, Biochemistry 15, 4521 (1976). [PubMed: 974073]

9. _ , ibid., p. 4529.

10. Chen LA, Dale RE, Roth S, Brand L, J. Biol. Chem 252, 2163 (1977). [PubMed: 849925]

11. Weber G,J. Chem. Phys 66, 4081 (1977).

12. Mantulin WW and Weber G, ibid., p. 4091.

13. Fluorescence polarization $(\mathrm{P})$ and anisotropy $(r)$ may be interconverted by using $\mathrm{P}=3 \mathrm{r} /(2+\mathrm{r})$. Fluorescence anisotropy is defined by $r=\frac{I_{\|}-I_{\|}}{I_{\|}+2 I_{\perp}}$

Where III and $\mathrm{I} \perp$, are the time-averaged values of $\mathrm{I} \|(\mathrm{t})$ and $\mathrm{I} \perp(\mathrm{t})$. The limiting anisotropy ro is related to the average angle $(\phi)$ to which torsional motions are restricted at times long compared to the fluorescence lifetime by $\mathrm{r} 0 / \mathrm{r} \infty=2 /(3 \cos 2 \phi-1)$. It is important to recognize that $\mathrm{r} \infty$. is a measure of the average angle, and not a measure of the maximum angle through which the fluorophore rotates.

14. Lakowicz JR and Prendergast FG, unpublished observations.

15. Weber G, Acta Phys. Pol, in press.

16. Spencer RD and Weber G, Ann. N.Y. Acad. Sci 158, 361 (1969).

17. _. J. Chem. Phys 52, 1654 (1970).

18. We thank the Freshwater Biological Research Foundation, and especially its founder, R. Gray, Sr., without whose assistance this work would not have been possible. We also thank D. Hogen for valuable technical assistance. The generous support of the American Heart Association, the Mayo Foundation, and the National Institutes of Health under grants ES GM 01238-OlAI (to J.R.L.) and CA 150 83-00 (to F.G.P.) is acknowledged. J.R.L. is an established investigator of the American Heart Association. Our special thanks are due to G. Weber for having supplied us with the theory of hindered rotations (as yet unpublished), and for his continuous generous support of this work. 


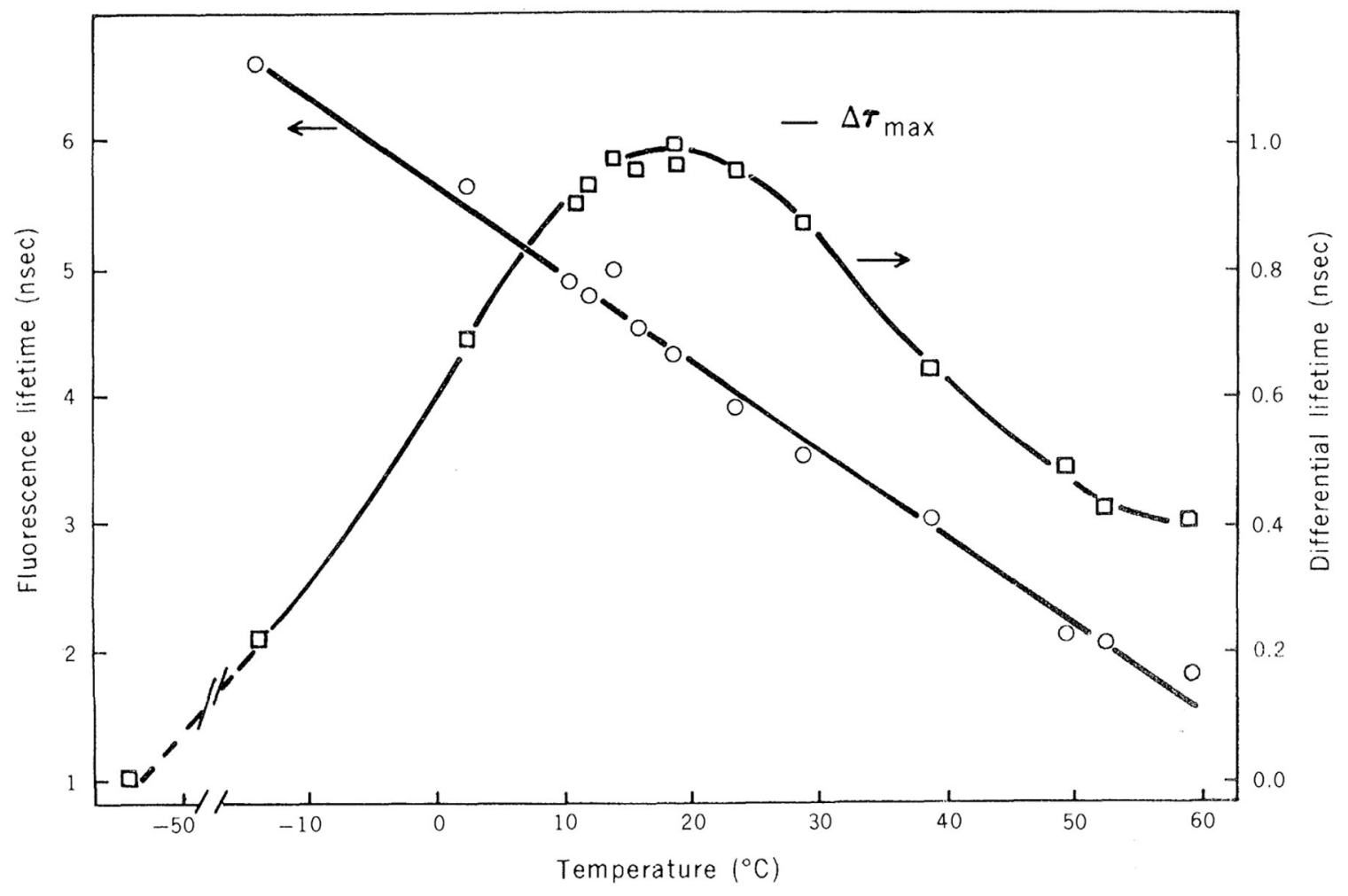

Fig. 1.

Fluorescence lifetimes and differential polarized phase lifetimes of DPH in propylene glycol. 


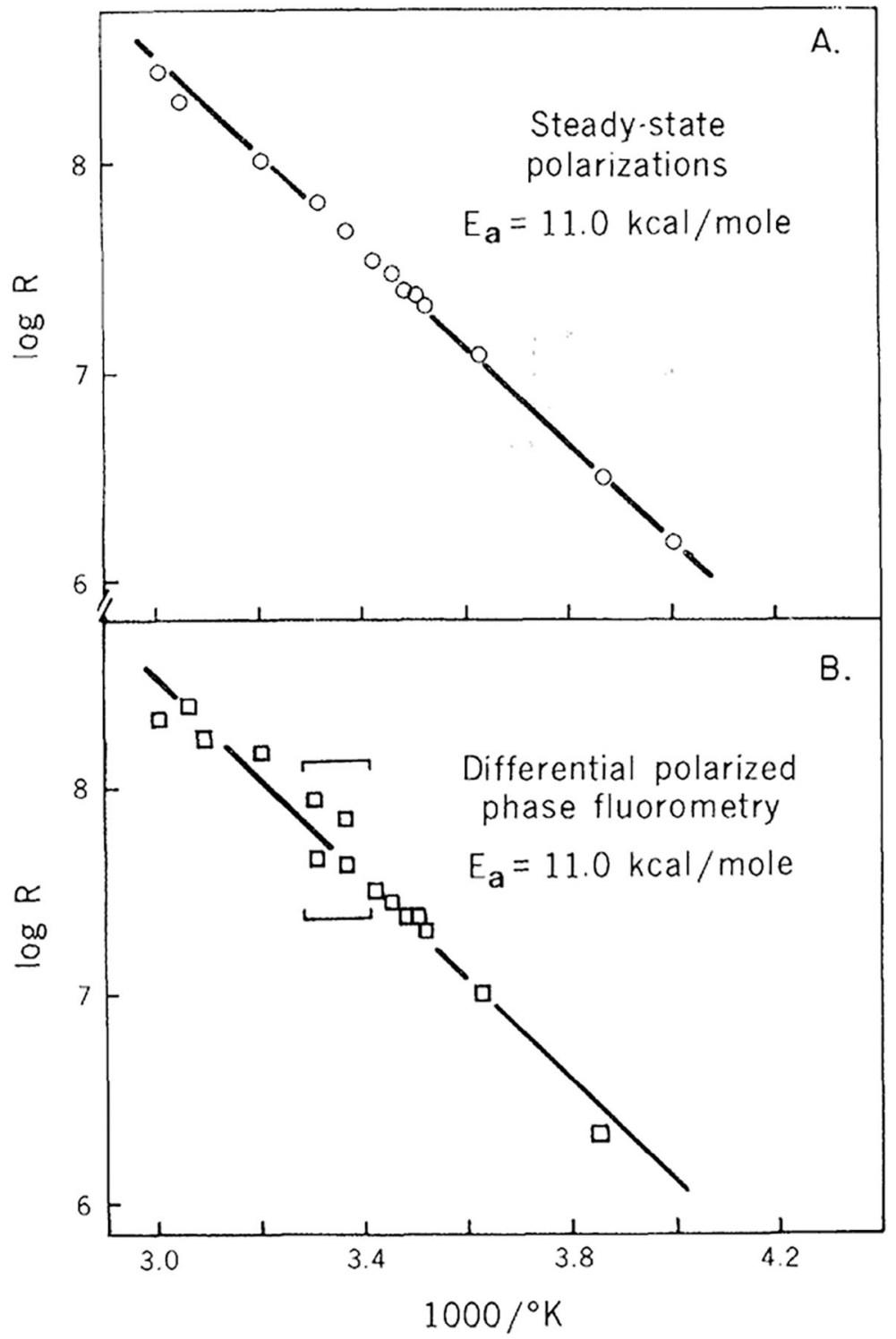

Fig. 2.

Rotational rate of DPH in propylene glycol. Rotational rates were calculated by both steadystate polarization mea surements (A) and differential polarized phase fluorometry (B). The duplicate points between the bars in (B) result from the two possible solutions to Eq. 1. Near $\Delta \tau=\Delta \tau_{\max }$ the choice of the proper solution is not clear. However, the value chosen is often unimportant since the rotational rates are similar. 


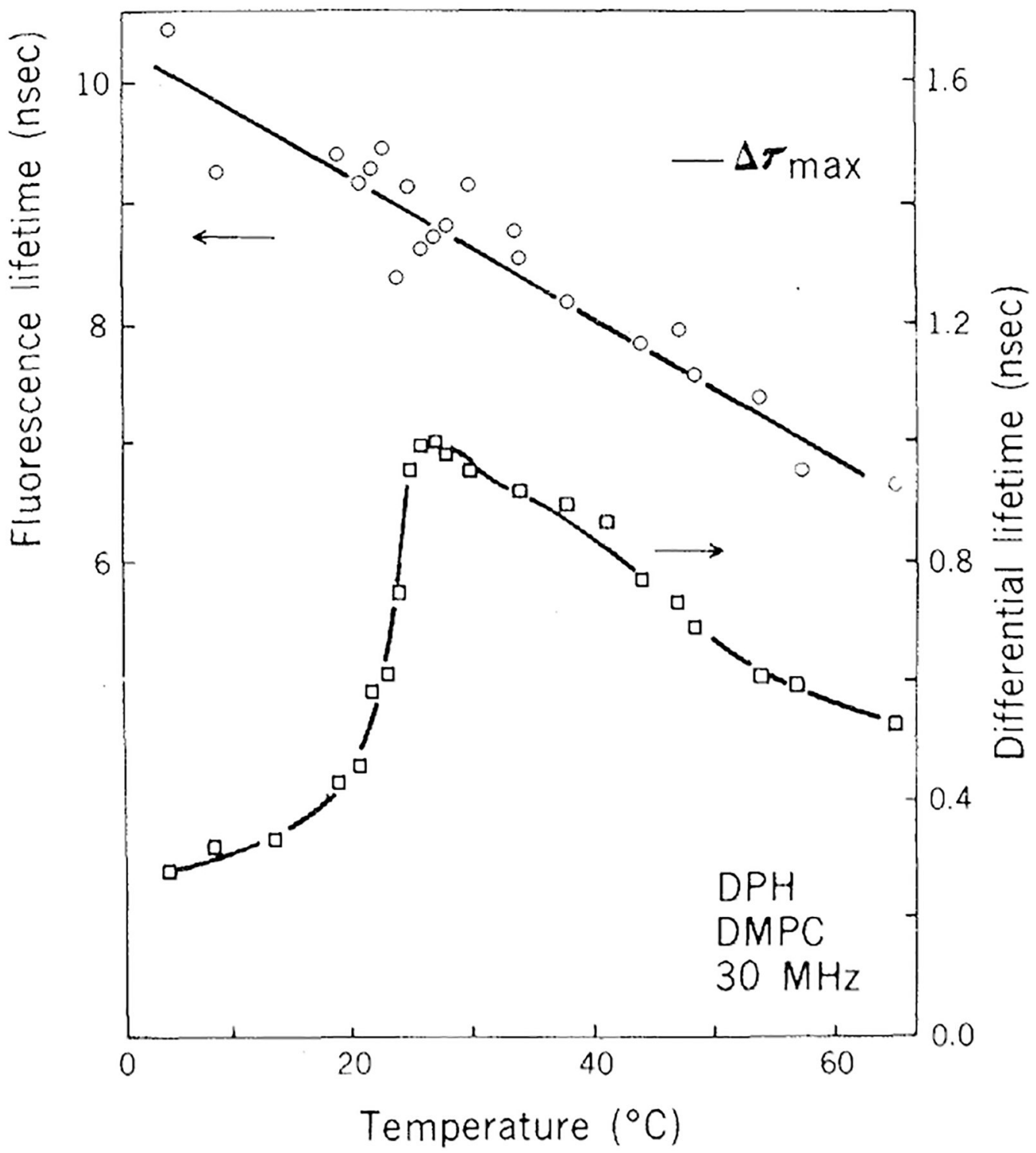

Fig. 3.

Fluorescence lifetimes (O) and differential polarized phase lifetimes ( $\square$ ) of DPH in DMPC vesicles. he $\Delta \tau_{\max }$ indicates the maximum differential lifetime predicted for a free isotropic rotator with $r_{0}=0.392$ and $\tau=8.7 \mathrm{nsec}$. 


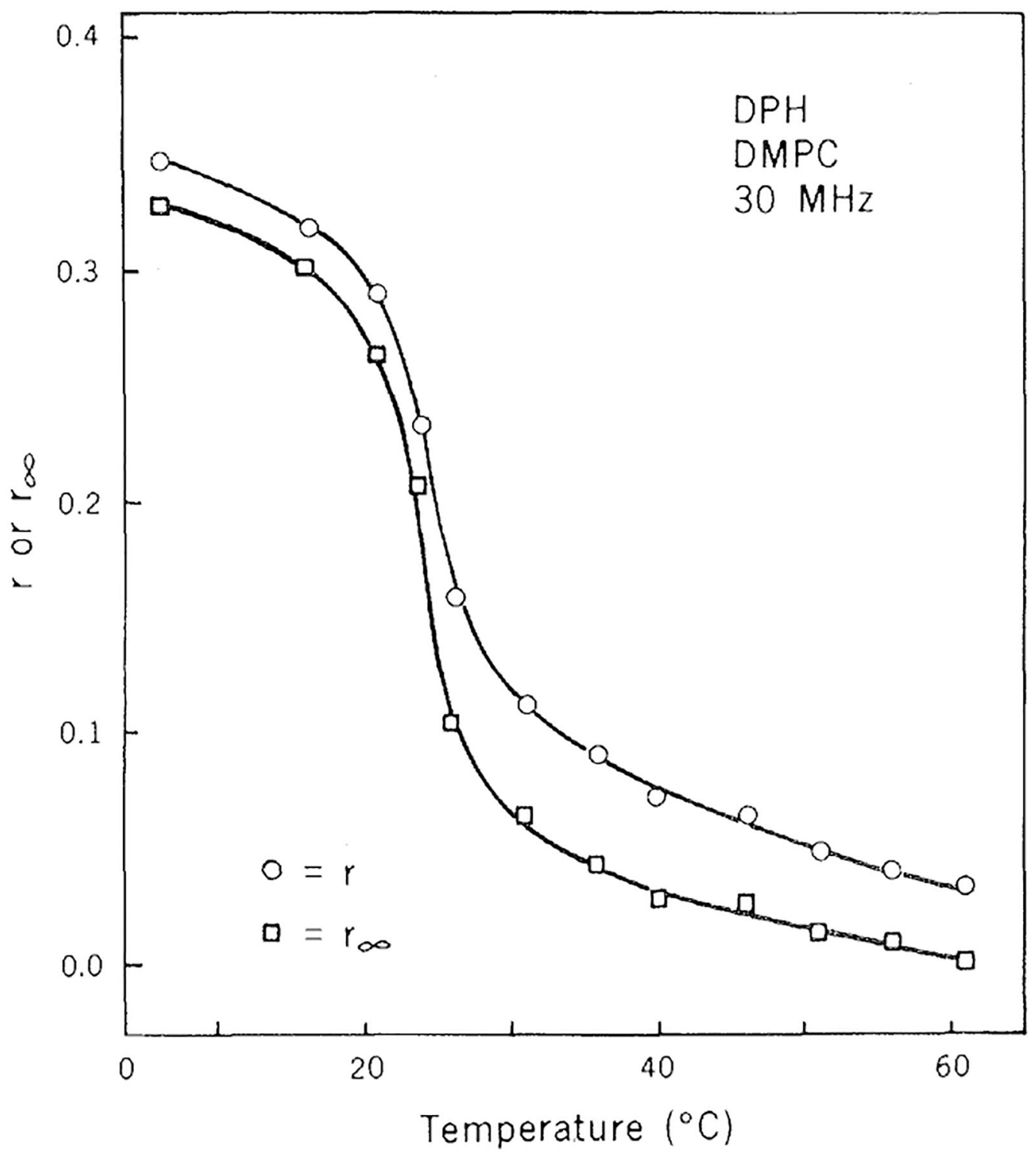

Fig. 4.

Limiting anisotropy values for DPH in DMPC vesicles. Limiting anisotropy values $(\square)$ were calculated from Eqs. 9 to 11, using data from Fig. 3 at regular temperature intervals, and from the steady-state anisotropy values $(\bigcirc)$. By using data read off Fig. 3, we effectively smoothed the data and minimized errors resulting from single data points. There are two solutions for $r_{\infty}$. at each point because Eq. 11 is quadratic. However, in each in stance one of the rotational rates was negative and thus inadmissible. 


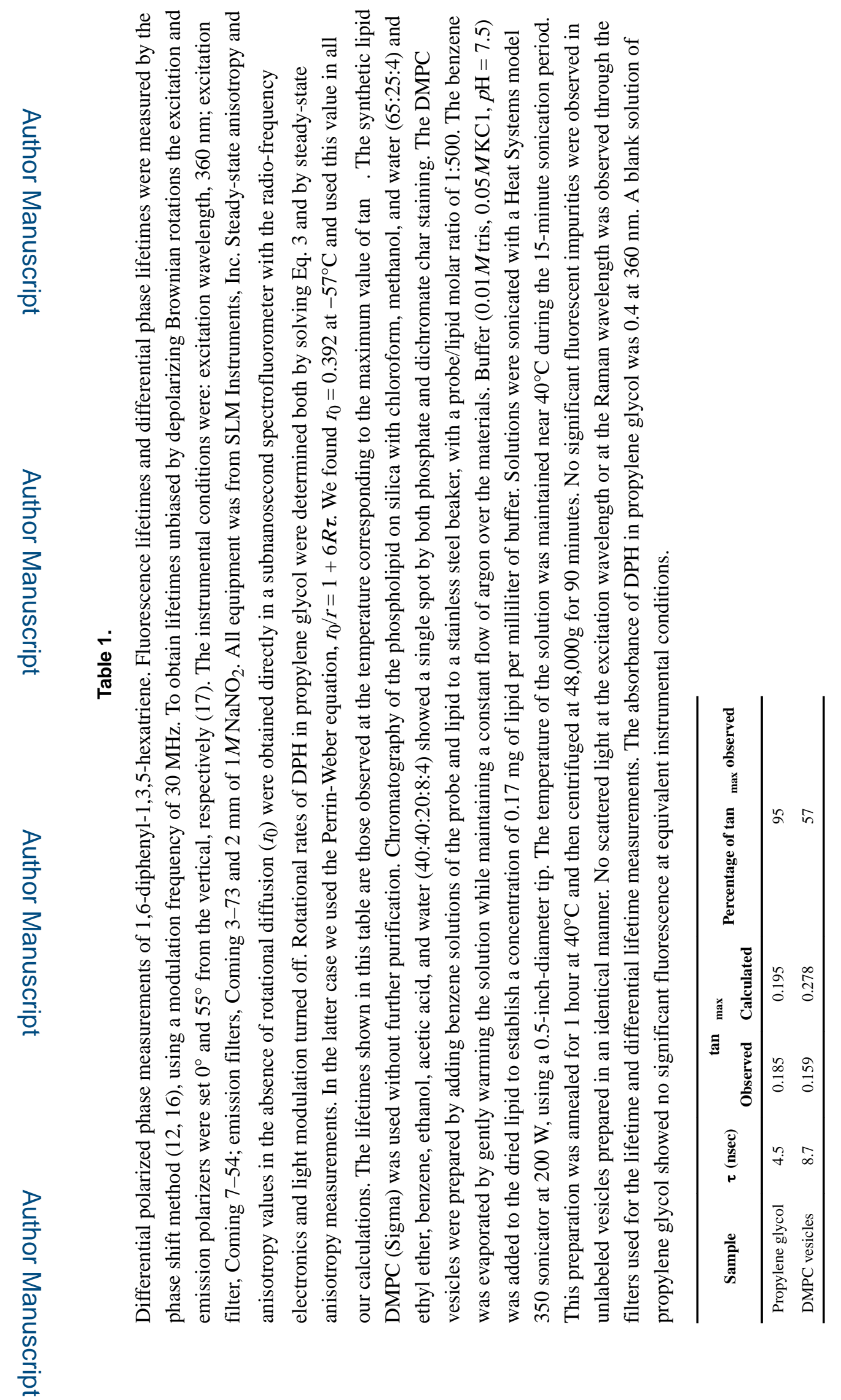

Science. Author manuscript; available in PMC 2019 December 04. 